\title{
Clinical Implications of Non-A-Type NPM1 and FLT3 Mutations in Patients with Normal Karyotype Acute Myeloid Leukemia
}

\author{
Borae G. Park ${ }^{a}$ Hyun-Sook Chi ${ }^{a}$ Seo-Jin Park ${ }^{c}$ Sook Kyoung Min ${ }^{a}$ \\ Seongsoo Jang ${ }^{a}$ Chan-Jeoung Park ${ }^{a}$ Dae-Young Kim ${ }^{b}$ Jung-Hee Lee ${ }^{b}$ \\ Je-Hwan Lee ${ }^{b}$ Kyoo-Hyung Lee ${ }^{b}$ \\ Departments of a Laboratory Medicine and ${ }^{\mathrm{b}}$ Internal Medicine, University of Ulsan College of Medicine, \\ Asan Medical Center, and ' Department of Laboratory Medicine, Yonsei University College of Medicine, \\ Seoul, South Korea
}

\section{Key Words}

Acute myeloid leukemia • FLT3 • Nucleophosmin •

Prognosis

\begin{abstract}
Mutations in the nucleophosmin (NPM1) and fms-like tyrosine kinase-3 (FLT3) genes are the most commonly observed mutations in patients with normal-karyotype acute myeloid leukemia (AML-NK). We analyzed the prognostic effects and interactions of these mutations in 201 AML-NK patients. NPM1 and FLT3 mutations were found in 38.3 and $24.9 \%$ of AML-NK patients, respectively. NPM1 mutations (NPM1mut), especially in patients without FLT3 mutations (FLT3mut), were associated with a favorable outcome. However, NPM1mut did not affect survival. FLT3mut tended to be associated with a poor survival outcome. FLT3mut showed no prognostic effects in patients with A-type NPM1mut. However, FLT3mut were associated with a significantly worse prognosis in patients with non-A-type NPM1mut. The prognostic interaction between the NPM1 and FLT3 mutations was significant in patients with non-A-type NPM1mut.
\end{abstract}

Copyright ๑ 2011 S. Karger AG, Base
(C) 2011 S. Karger AG, Base

$0001-5792 / 12 / 1272-0063 \$ 38.00 / 0$

Fax +41613061234 E-Mail karger@karger.ch www.karger.com

\section{Introduction}

New molecular markers have been used to determine the prognosis of patients with acute myeloid leukemia (AML), especially patients with normal-karyotype AML (AML-NK). These patients constitute approximately $40 \%$ of patients with AML and are regarded as an intermediaterisk group $[1,2]$. In addition, these patients harbor specific mutations in genes that serve as prognostic markers for survival outcomes after adequate therapy $[1,2]$. Prognostically important mutations in patients with AML-NK include mutations in the nucleophosmin (NPM1), fmsrelated tyrosine kinase-3 (FLT3) and CCAAT/enhancer binding protein $\alpha(C E B P A)$ genes [3]. For example, NPM1 mutations (NPM1mut) are observed in $50-60 \%$ of patients with AML-NK and are included in the 2008 World Health Organization classification of myeloid neoplasms as provisional entities [3]. In contrast, FLT3 mutations (FLT3mut) are not included as defining criteria for distinct entities because they often accompany other genetic abnormalities. However, FLT3 internal tandem duplications (ITD) are the second most common mutation in patients with AML-NK [3-5]. NPM1mut are associated with a better patient prognosis, whereas FLT3-ITD mutations (FLT3-ITDmut) are associated with shorter disease-free survival and overall survival (OS) [4, 5]. Approximately

Hyun-Sook Chi, MD, Department of Laboratory Medicine

University of Ulsan College of Medicine, Asan Medical Center

86, Asanbyeongwon-gil, Songpa-gu

Seoul 138-736 (South Korea)

Tel. +82 23010 4502,E-Mail hschi@amc.seoul.kr 
Table 1. Clinical and demographic characteristics of patients with AML-NK

\begin{tabular}{|c|c|c|c|c|c|c|}
\hline & NPM1wt & $\begin{array}{l}\text { A-type } \\
\text { NPM1mut }\end{array}$ & $\begin{array}{l}\text { Non-A-type } \\
\text { NPM1mut }\end{array}$ & Total & $\mathrm{p}^{\mathrm{a}}$ & $\mathrm{p}^{\mathrm{b}}$ \\
\hline Patients & 124 & 61 & 16 & 201 & & \\
\hline Age, years & $49.9 \pm 14.7$ & $54.0 \pm 15.0$ & $52.6 \pm 14.6$ & $51.4 \pm 14.9$ & 0.075 & 0.514 \\
\hline Sex & & & & & 0.142 & 0.099 \\
\hline Male & $68(54.8)$ & $24(39.3)$ & $10(62.5)$ & $102(50.7)$ & & \\
\hline Female & $56(45.2)$ & $37(60.7)$ & $6(37.5)$ & $99(49.3)$ & & \\
\hline FAB subtype & & & & & 0.068 & 0.586 \\
\hline M0 & $3(2.4)$ & $1(1.6)$ & 0 & $4(2.0)$ & & \\
\hline M1 & $26(21.0)$ & $18(29.5)$ & $7(43.8)$ & $51(25.4)$ & & \\
\hline M2 & $39(31.5)$ & $19(31.1)$ & $1(6.3)$ & $59(29.4)$ & & \\
\hline M4 & $16(12.9)$ & $13(21.3)$ & $3(18.8)$ & $32(15.9)$ & & \\
\hline M5 & $3(2.4)$ & $5(8.2)$ & 0 & $8(4.0)$ & & \\
\hline M6 & $7(5.6)$ & $1(1.6)$ & 0 & $8(4.0)$ & & \\
\hline M7 & $5(4.0)$ & $2(3.3)$ & $1(6.3)$ & $8(4.0)$ & & \\
\hline M8 & $1(0.8)$ & 0 & 0 & $1(0.5)$ & & \\
\hline AML-MRC & $22(17.7)$ & $2(3.3)$ & $4(25.0)$ & $28(13.9)$ & & \\
\hline tAML & $2(1.6)$ & 0 & 0 & $2(1.0)$ & & \\
\hline $\mathrm{WBC}, \times 10^{9} / 1$ & $46.98 \pm 77.75$ & $49.23 \pm 60.25$ & $38.60 \pm 46.51$ & $46.99 \pm 70.54$ & 0.107 & 0.490 \\
\hline $\mathrm{Hb}, \mathrm{g} / \mathrm{dl}$ & $8.8 \pm 1.9$ & $8.8 \pm 1.4$ & $9.3 \pm 1.4$ & $8.8 \pm 1.7$ & 0.451 & 0.173 \\
\hline Platelets, $\times 10^{9} / 1$ & $71.5 \pm 64.1$ & $83.5 \pm 71.4$ & $79.2 \pm 72.4$ & $75.7 \pm 66.9$ & 0.228 & 0.589 \\
\hline BM blasts, $\%$ & $53.2 \pm 22.9$ & $66.7 \pm 20.5$ & $42.3 \pm 40.0$ & $43.5 \pm 35.6$ & 0.000 & 0.594 \\
\hline FLT3mut & $23(18.5)$ & $23(37.7)$ & $4(25.0)$ & $50(24.9)$ & 0.009 & 0.346 \\
\hline FLT3-ITDmut & 22 & 20 & 3 & 45 & & \\
\hline FLT3-TKDmut & 1 & 3 & 1 & 5 & & \\
\hline
\end{tabular}

Values represent either numbers (percentages) or means \pm standard deviation. FAB = French-American-British classification; AML-MRC $=$ AML with myelodysplasia-related change; tAML $=$ treatment-related AML.

${ }^{\text {a }}$ NPM1wt versus NPM1mut. ${ }^{\mathrm{b}}$ A-type versus non-A-type NPM1mut.

$80 \%$ of NPM1mut are A-type (tandem duplication of TCTG) [6], whereas the remaining $20 \%$ are non-A-type (exon 12 mutations), which have been associated with poor prognosis [7]. Mutations in the FLT3 tyrosine kinase domain (TKD) have been observed in approximately $4-7 \%$ of patients with AML; however, little is known about the clinical impact of these FLT3-TKD mutations [8-10].

It has not been determined whether or not these mutations exert positive or negative effects on prognosis after treatment [11]. Several Japanese and Korean studies have described the prognostic significance of NPM1mut and FLT3mut in various clinical settings. However, these studies did not focus on AML-NK [7, 10, 12, 13]. The prognostic effects of these mutations in AML-NK have been emphasized. Therefore, to further investigate the effects of these mutations on survival in AML-NK patients, we assessed the frequencies and prognostic effects of NPM1mut and FLT3mut, including non-A-type NPM1 and FLT3-TKD mutations, and their interactions in AML-NK patients.

\section{Patients and Methods}

\section{Patients}

Our patient population comprised 201 adults (102 men, 99 women) who were aged 15 years or older (median age 53 years, range $15-81$ years, mean \pm SD $51.4 \pm 14.9$ years) and had been newly diagnosed with AML-NK at the Asan Medical Center between January 2002 and March 2010 (table 1). Of these 201 patients, 153 (76.1\%; 77 men and 76 women; median age 48 years, range $15-79$ years, mean \pm SD $48.2 \pm 14.1$ years) received standard induction chemotherapy consisting of 7 days of cytarabine at 200 $\mathrm{mg} / \mathrm{m}^{2} /$ day $\left(100 \mathrm{mg} / \mathrm{m}^{2} /\right.$ day for patients aged $>60$ years $)$ and 3 days of daunorubicin at $45 \mathrm{mg} / \mathrm{m}^{2} /$ day. Patients who failed to achieve complete remission (CR) but attained partial remission received a second, identical cycle of induction chemotherapy. Consolidation chemotherapy was followed by anthracycline and cytarabine (or cytarabine and daunorubicin for patients aged $>60$ years) according to the standard regimen of our institution [14]. CR was defined as the presence of $<5 \%$ blast cells with more than $20 \%$ cellularity in a standardized bone marrow (BM) aspirate sample after the first or second course of induction chemotherapy according to our institution's criteria [15]. The study protocol was approved by our institutional review board, and all patients provided written informed consent for additional genetic analyses at diagnosis. 
Table 2. List of NPM1 exon 12 mutations that were observed in our patients with AML-NK

\begin{tabular}{llll}
\hline \multirow{2}{*}{$\begin{array}{l}\text { Type of } \\
\text { mutation }^{\text {a }}\end{array}$} & $\begin{array}{l}\text { Nucleotide sequence } \\
\text { of insertion at c.860_863 }\end{array}$ & Patients \\
\cline { 3 - 4 } & & $\mathrm{n}$ & $\%$ \\
\hline $\begin{array}{l}\text { A type } \\
\text { Non-A type }\end{array}$ & TCTG & 61 & 79.2 \\
B & CATG & 5 & 6.5 \\
D & CCTG & 3 & 3.9 \\
Qm & TCGG & 2 & 2.6 \\
Nm & CCAG & 1 & 1.3 \\
C & CGTG & 1 & 1.3 \\
4 & CTTG & 1 & 1.3 \\
NR & TCGA & 1 & 1.3 \\
NR & AGGC & 1 & 1.3 \\
NR & CCGC & 1 & 1.3 \\
\hline
\end{tabular}

$\mathrm{NR}=$ Not reported.

a The type of mutation was defined according to previously published data [17].

Analyses of NPM1mut and FLT3mut

$\mathrm{BM}$ samples that were obtained at diagnosis were retrospectively analyzed for NPM1mut and FLT3mut. Genomic DNA was extracted from BM smears or cell pellets using QIAamp DNA Blood Mini Kits (Qiagen, Hilden, Germany).

The analysis of NPM1mut was performed using fragment analysis as previously described [6]. Genomic DNA was amplified using PCR analysis. The products were subjected to capillary electrophoresis for exact size analysis using an ABI 3100 genetic analyzer (Applied Biosystems, Foster City, Calif., USA) and GeneScan Analysis software (Applied Biosystems). In samples showing an additional peak, which indicated the presence of the 4-bp insertion, the NPM1 exon 12 was amplified and the products were directly sequenced.

The presence of FLT3-ITD and TKD mutations was determined using multiplex PCR analysis with Seeplex FLT3 Genotyping Kits (Seegene, Korea).

\section{Statistical Analysis}

Fisher's exact test was used to compare categorical variables between two groups, and the Mann-Whitney U test was used to compare continuous variables. The Kruskal-Wallis test was used to compare variables among three groups. Relapse-free survival (RFS) was calculated from the time of CR until relapse, death or the end of the study. OS was measured from the date of the first diagnosis to death from any cause during the course of this study. Nonparametric survival curves for RFS and OS were calculated using the Kaplan-Meier method and compared using the log-rank test. Multivariate Cox proportional hazard regression analysis was used to identify independent prognostic factors for RFS and OS. $p<0.05$ was considered statistically significant. All statistical analyses were performed using SPSS 12.0 software (SPSS, Chicago, Ill., USA).

\section{Results}

\section{Incidence of NPM1mut and FLT3mut in AML-NK Patients}

The clinical characteristics of our 201 adult patients with AML-NK are shown in table 1 . Of these 201 patients, 77 (38.3\%) exhibited NPM1mut. When grouped according to patient age, the incidence of NPM1mut was $28.6 \%(14 / 49)$ in patients aged $<40$ years, $42.1 \%(37 / 88)$ in patients aged $41-60$ years and $40.6 \%(26 / 64)$ in patients aged $>60$ years. NPM1, especially the A-type NPM1mut, was more common in female patients ( $\mathrm{p}=$ 0.142). The incidence of NPM1mut was higher in patients with AML-M1, -M4 and -M5 than in patients with other types of leukemia $(\mathrm{p}=0.068)$. The complete blood cell counts at diagnosis were not significantly different between patients with and without NPM1mut. However, BM blasts were significantly increased in patients with NPM1mut ( $\mathrm{p}=0.000$; table 1$)$. The most common NPM1mut was the type A mutation ( $\mathrm{n}=61,79.2 \%$ ), which is a duplication of TCTG at position c.860_863 (NCBI accession number NM_002520.5) [16, 17]. In addition, 5 patients $(6.5 \%)$ had type B mutations, 3 (3.9\%) had type D, 2 (2.6\%) had type Qm and 6 (7.8\%) had other types of NPM1mut (table 2).

We also detected FLT3mut in 50 patients $(24.9 \%)$ and showed that 45 patients (22.4\%) exhibited FLT3-ITDmut, whereas 5 patients $(2.5 \%)$ displayed FLT3-TKD mutations. The incidence of FLT3mut, regardless of the type of mutation, was significantly higher in patients with NPM1mut than in patients without NPM1mut ( $\mathrm{p}=0.009$; table 1).

\section{Prognostic Impact of NPM1mut and FLT3-ITDmut}

Of the 153 patients who received standard induction chemotherapy, 121 patients (79.1\%) achieved CR. The CR rate was significantly higher in patients with NPM1mut than in patients with wild-type NPM1 (NPM1wt; 86.2 vs. $74.7 \%, p=0.007$; table 3 ). Of the patients who achieved CR, patients with NPM1mut had a lower relapse rate and a higher survival rate than patients without NPM1mut (relapse rate: 32.8 vs. $41.1 \%, \mathrm{p}=0.081$; survival rate: 56.9 vs. $44.2 \%, \mathrm{p}=0.129)$. However, these differences were not statistically significant (table 3). NPM1mut were not independently associated with RFS or OS. Of the 153 patients who received standard induction chemotherapy, 75 (49.0\%) underwent hematopoietic stem cell transplantation (HSCT), regardless of whether or not they had NPM1mut. Most patients who received allogeneic HSCT, regardless of donor type, underwent re- 
Table 3. Clinical characteristics and treatment outcomes based on NPM1mut status

\begin{tabular}{|c|c|c|c|c|c|c|}
\hline & NPM1wt & $\begin{array}{l}\text { A-type } \\
\text { NPM1mut }\end{array}$ & $\begin{array}{l}\text { Non-A-type } \\
\text { NPM1mut }\end{array}$ & Total & $\mathrm{p}^{\mathrm{a}}$ & $\mathrm{p}^{\mathrm{b}}$ \\
\hline Patients & 95 & 45 & 13 & 153 & & \\
\hline Age, years & $47.0 \pm 14.1$ & $51.1 \pm 14.4$ & $49.0 \pm 12.9$ & $48.2 \pm 14.1$ & 0.061 & 0.406 \\
\hline \multicolumn{7}{|l|}{ Sex } \\
\hline Male & $50(52.6)$ & $20(44.4)$ & $7(53.8)$ & $77(50.3)$ & 0.467 & 0.553 \\
\hline Female & $45(47.4)$ & $25(55.6)$ & $6(46.2)$ & $76(49.7)$ & & \\
\hline FLT3mut & $20(21.1)$ & $18(40.0)$ & $4(30.8)$ & $42(27.5)$ & 0.024 & 0.549 \\
\hline FLT3-ITDmut & 20 & 15 & 3 & 38 & & \\
\hline FLT3-TKDmut & 0 & 3 & 1 & 4 & & \\
\hline CR & $71(74.7)$ & $38(84.4)$ & $12(92.3)$ & $121(79.1)$ & 0.007 & 0.219 \\
\hline HSCT & $49(51.6)$ & $20(44.4)$ & $6(46.2)$ & $75(49.0)$ & 0.419 & 0.914 \\
\hline Relapse & $39(41.1)$ & $16(35.6)$ & $3(23.1)$ & $58(37.9)$ & 0.081 & 0.320 \\
\hline Mean RFS, months & $17.67 \pm 24.07$ & $22.31 \pm 22.44$ & $24.47 \pm 24.49$ & $19.61 \pm 23.62$ & 0.073 & 0.881 \\
\hline Death & $53(55.8)$ & $19(42.2)$ & $6(46.2)$ & $78(51.0)$ & 0.129 & 0.803 \\
\hline OS, months & $24.38 \pm 23.93$ & $26.25 \pm 22.24$ & $29.45 \pm 27.53$ & $25.36 \pm 23.65$ & 0.591 & 0.993 \\
\hline
\end{tabular}

duced-intensity conditioning therapy consisting of busulfan, fludarabine and antithymocyte globulin according to the standard regimen of our institution [18].

Based on their NPM1 and FLT3-ITDmut status, patients were divided into 4 groups. Patients with NPM1mut but without FLT3-ITDmut (NPM1mut/FLT3-ITDwt) had the highest $\mathrm{CR}$ rate $(92.5 \%$; table 4$)$ and significantly longer RFS ( $p=0.004)$ and OS ( $p=0.055$; fig. 1$)$ than the other groups. Multivariate analysis using a Cox regression model showed that HSCT was the only factor that was independently associated with longer RFS $(p=0.000)$ and $O S(p=0.000)$. No significant differences among the 4 groups were detected after adjustment for HSCT (table 5). In each group, the OS rate was higher in patients who underwent HSCT than in those who did not (63.4 vs. 26.5\%, $\mathrm{p}=0.000$, in NPM1wt/FLT3-ITDwt patients; 88.9 vs. $40.9 \%, \mathrm{p}=0.007$, in NPM1mut/FLT3-ITDwt patients; 62.5 vs. $16.7 \%, \mathrm{p}=0.105$, in NPM1wt/FLT3-ITDmut patients; 75.0 vs. $20.0 \%, \mathrm{p}=0.000$, in NPM1mut/FLT3ITDmut patients).

\section{Prognostic Impact and Interaction according to the}

Type of Mutations

We found that the CR rate tended to be higher in patients with non-A-type NPM1mut than in patients with A-type NPM1mut (92.3 vs. $84.4 \%$; $\mathrm{p}=0.238$ ), regardless of the FLT3mut status (table 4). Moreover, the CR rate
Table 4. Complete remission rate based on risk factors

\begin{tabular}{llll}
\hline Risk factor & $\begin{array}{l}\text { Achieved } \\
\text { CR, } \mathrm{n}\end{array}$ & $\begin{array}{l}\text { Total } \\
\mathrm{n}\end{array}$ & $\begin{array}{l}\text { CR rate } \\
\%\end{array}$ \\
\hline NPM1mut/FLT3-ITDwt & 37 & 40 & 92.5 \\
NPM1wt/FLT3-ITDmut & 12 & 20 & 60.0 \\
NPM1mut/FLT3-ITDmut & 13 & 18 & 72.2 \\
NPM1wt/FLT3-ITDwt & 59 & 75 & 78.7 \\
A-type NPM1mut & 38 & 45 & 84.4 \\
Non-A-type NPM1mut & 12 & 13 & 92.3 \\
FLT3-ITDmut & 25 & 38 & 65.8 \\
FLT3-TKDmut & 3 & 4 & 75.0 \\
\hline
\end{tabular}

$\mathrm{CR}=$ Complete remission.

tended to be lower in patients with FLT3-ITDmut than in those with FLT3-TKD mutations $(65.8$ vs. $75.0 \%$, p = 0.359 ; table 4). However, these differences were not statistically significant. Kaplan-Meier survival analyses showed no significant differences in RFS ( $\mathrm{p}=0.459)$ or OS ( $\mathrm{p}=0.847)$ between patients with A-type NPM1mut and those with non-A-type NPM1mut (fig. 2). In addition, no differences were observed in RFS $(\mathrm{p}=0.674)$ or OS ( $\mathrm{p}=0.897$ ) between patients with FLT3-ITDmut and those with FLT3-TKD mutations. A multivariate analysis 
Table 5. Cox proportional hazard models for RFS and OS in AML-NK patients based on NPM1mut and FLT3-ITDmut

\begin{tabular}{llll}
\hline $\begin{array}{l}\text { Risk factor by survival } \\
\text { end points }\end{array}$ & HR & $95 \%$ CI & $\mathrm{p}$ \\
\hline RFS & & & \\
HSCT & 0.302 & $0.172-0.531$ & 0.000 \\
NPM1mut/FLT3-ITDwt & 0.446 & $0.176-1.131$ & 0.089 \\
NPM1wt/FLT3-ITDmut & 2.019 & $0.764-5.333$ & 0.156 \\
NPM1mut/FLT3-ITDmut & 1.634 & $0.533-5.009$ & 0.390 \\
NPM1wt/FLT3-ITDwt & 0.981 & $0.393-2.452$ & 0.968 \\
OS & & & \\
HSCT & 0.229 & $0.136-0.384$ & 0.000 \\
NPM1mut/FLT3-ITDwt & 0.611 & $0.242-1.540$ & 0.296 \\
NPM1wt/FLT3-ITDmut & 1.417 & $0.549-3.657$ & 0.472 \\
NPM1mut/FLT3-ITDmut & 1.594 & $0.530-4.792$ & 0.407 \\
NPM1wt/FLT3-ITDwt & 1.102 & $0.451-2.694$ & 0.832 \\
RFS & & & \\
HSCT & 0.291 & $0.168-0.503$ & 0.000 \\
A-type NPM1mut & 0.533 & $0.289-0.985$ & 0.045 \\
Non-A-type NPM1mut & 0.426 & $0.131-1.392$ & 0.158 \\
FLT3-ITDmut & 3.025 & $1.687-5.423$ & 0.000 \\
FLT3-TKDmut & 1.159 & $0.259-5.192$ & 0.847 \\
OS & & & \\
HSCT & 0.234 & $0.141-0.388$ & 0.000 \\
A-type NPM1mut & 0.609 & $0.352-1.055$ & 0.077 \\
Non-A-type NPM1mut & 0.842 & $0.357-1.985$ & 0.695 \\
FLT3-ITDmut & 1.935 & $1.176-3.182$ & 0.009 \\
FLT3-TKDmut & 1.180 & $0.346-4.026$ & 0.792 \\
\hline
\end{tabular}

$\mathrm{HR}=$ Hazard ratio; $\mathrm{CI}=$ confidence interval.

using a Cox regression model showed that FLT3-ITDmut were independently associated with significantly shorter RFS $(\mathrm{p}=0.001)$ and OS $(\mathrm{p}=0.010)$ after adjustment for HSCT (table 5). A-type NPM1mut and non-A-type NPM1mut were associated with longer RFS and OS. FLT3-TKD mutations were associated with shorter RFS and OS, although these differences were not statistically significant (table 5).

Only 56 patients were enrolled in this study, and the prognostic study for the interaction between NPM1mut and FLT3mut showed weaker effects because of the small number of patients. In the 42 patients with A-type NPM1mut, the presence of FLT3mut, including FLT3TKD mutations, did not significantly affect the RFS ( $\mathrm{p}=$ $0.081)$ or OS ( $\mathrm{p}=0.210$; fig. 2 ). In the 14 patients with nonA-type NPM1mut, the RFS ( $\mathrm{p}=0.001)$ and OS $(\mathrm{p}=0.062)$ tended to decrease in patients with FLT3mut compared with patients without FLT3mut (fig. 2).

NPM1 and FLT3 Mutations in AML
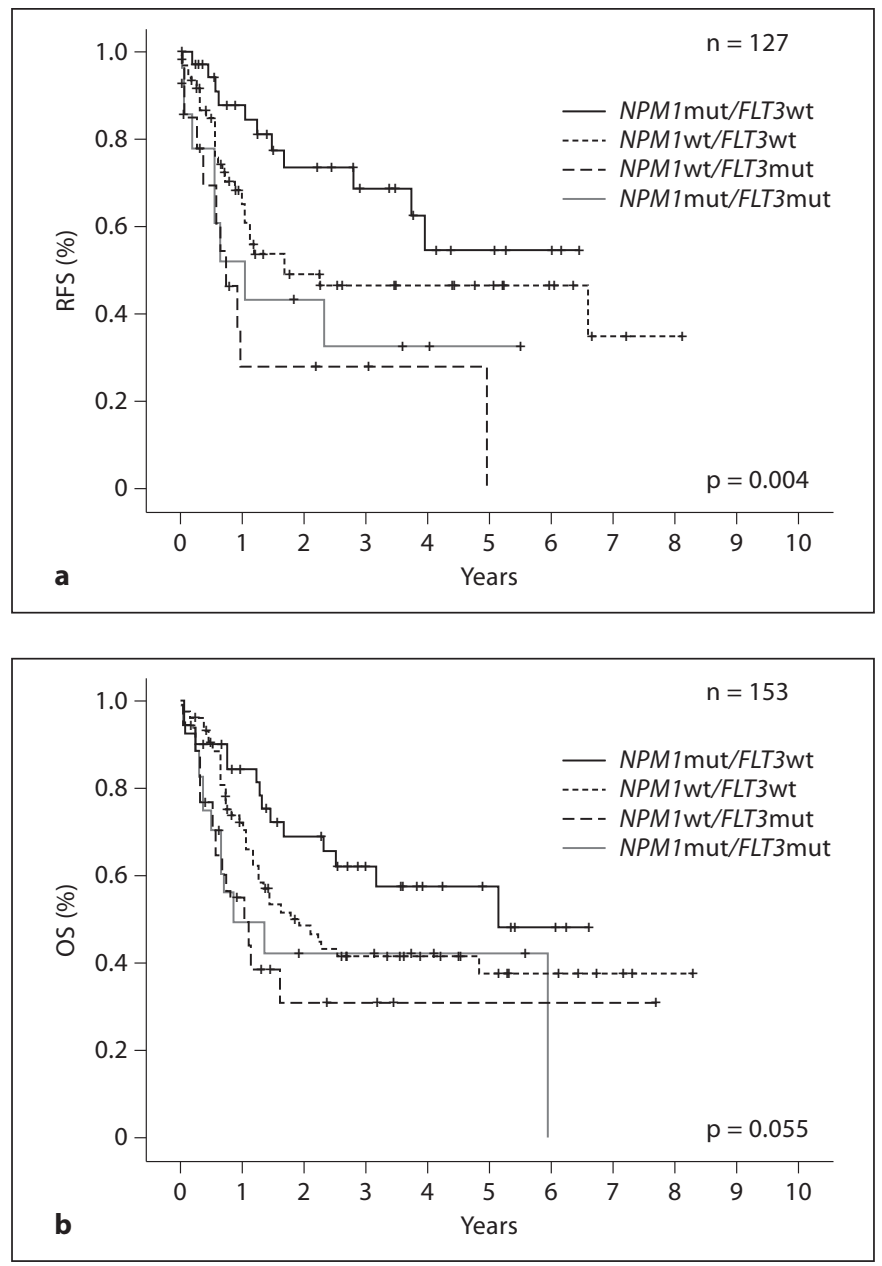

Fig. 1. a RFS in patients with AML-NK according to NPM1 and FLT3-ITDmut. b OS of patients with AML-NK according to NPM1 and FLT3-ITDmut. Patients with NPM1mut but without FLT3-ITDmut had significantly better RFS and OS than other patient groups. NPM1mut/FLT3wt: patients with NPM1mut without FLT3-ITDmut; NPM1mut/FLT3mut: patients with both NPM1 and FLT3-ITDmut; NPM1wt/FLT3wt: patients with neither NPM1 nor FLT3-ITDmut; NPM1wt/FLT3mut: patients with FLT3-ITDmut without NPM1mut.

\section{Discussion}

We retrospectively investigated the incidence and clinical impact of NPM1 and FLT3 mutations in 201 Korean patients with AML-NK. We found that among these patients, $38.3 \%$ had NPM1mut and $24.9 \%$ had FLT3mut, which are similar findings to incidences that have been previously reported in Asian populations [8, 19]. Several Japanese studies observed a lower incidence of NPM1mut than in our study $[12,13]$. However, those studies evaluated AML with or without abnormal karyotype. Al- 

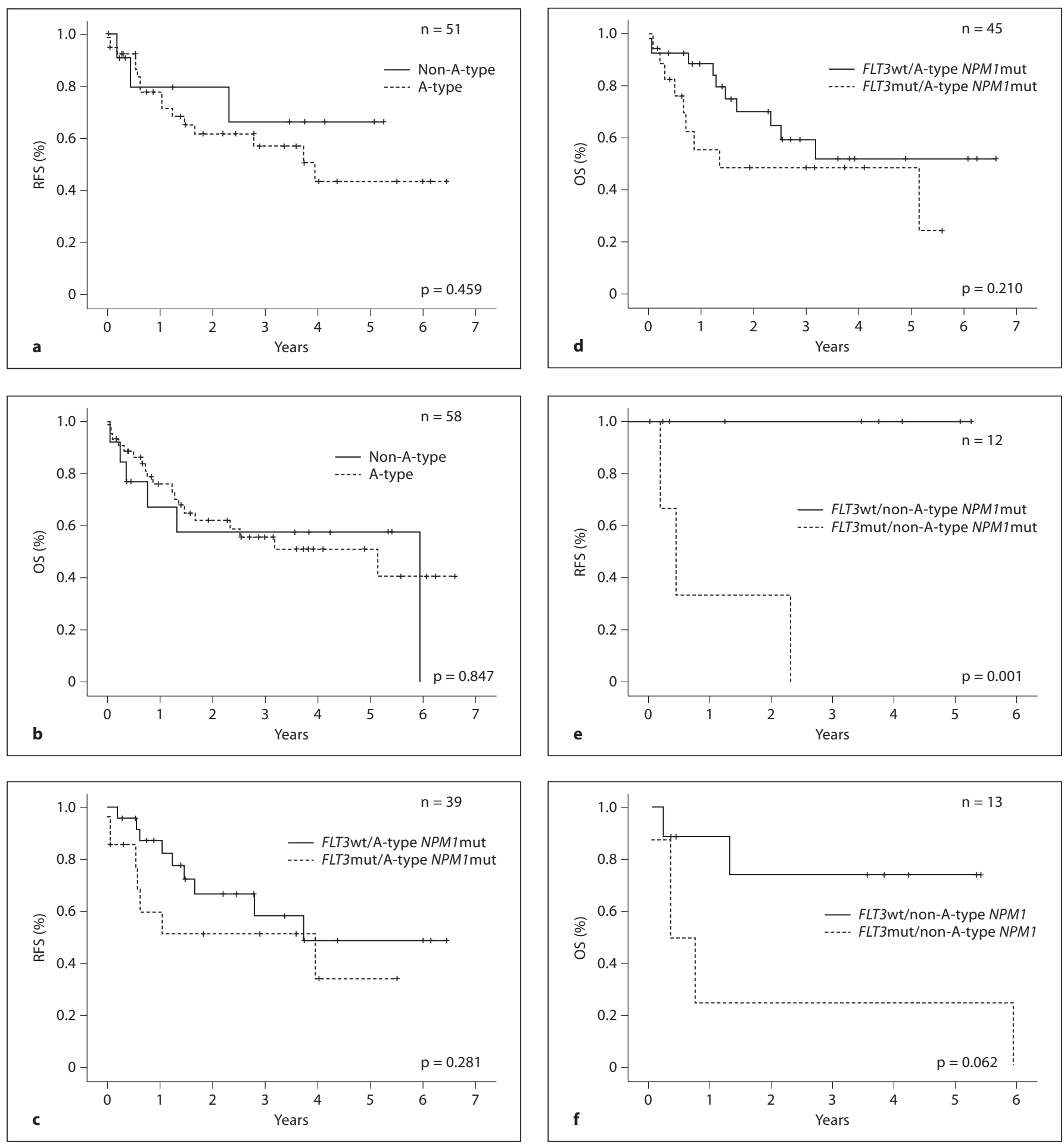

Fig. 2. a RFS of patients with NPM1mut according to the type of NPM1mut. $\mathbf{b}$ OS of patients with NPM1mut according to the type of NPM1mut. No significant differences in survival outcomes were detected between patients with A-type and non-A-type NPM1mut. c RFS of patients with A-type NPM1mut according to FLT3mut. d OS of patients with A-type NPM1mut according to

FLT3mut. No significant differences were detected in survival between patients with FLT3wt and FLT3mut. e RFS of patients with non-A-type NPM1mut according to FLT3mut. The RFS was significantly increased in patients with FLT3wt compared to patients with FLT3mut. $\mathbf{f}$ OS of patients with non-A-type NPM1 mut according to FLT3mut. 
though NPM1mut was detected in AML with an abnormal karyotype, its prevalence was more frequent in AMLNK. NPM1mut has a favorable prognosis in AML-NK [5, 20,21]. Therefore, a prognostic study for NPM1mut must focus on AML-NK. However, most prognostic studies for NPM1mut in Asians have been performed in AML with or without abnormal karyotypes [12, 13]. Our study is therefore advantageous from this point of view.

NPM1mut is more common in older than in younger patients [19]. We showed that the incidence of NPM1mut was lower in adults who were aged $<40$ years but similar in patients who were aged $40-60$ and $>60$ years. In agreement with previous findings, we found that NPM1mut was significantly associated with an increased percentage of BM blasts [19]. However, the increase in BM blasts was not associated with a difference in patient prognosis (data not shown).

NPM1 and FLT3 mutations have been suggested to be mechanistically linked $[16,22]$. Patients with FLT3mut are more likely to have NPM1mut $[8,9]$. We found that FLT3-ITD and FLT3-TKD mutations were more common in patients with NPM1mut than in those with NPM1wt. Although 5 of 201 patients (2.5\%) had the FLT3-TKD mutation, 4 of these 5 patients (80.0\%) also carried NPM1mut. The interaction between FLT3mut and NPM1mut may have prognostic impact because patients with NPM1mut/FLT3-ITDwt had significantly longer RFS and tended to have longer OS than patients with one of the other three genotypes. Moreover, the NPM1mut/FLT3-ITDwt combination showed a favorable hazard ratio. However, NPM1mut/FLT3-ITDwt was not a significant prognostic factor in the Cox regression model. Recent studies have demonstrated that patients with NPM1mut but without FLT3-ITD have a better prognosis $[23,24]$. However, most studies on the interaction between NPM1mut and FLT3mut in AML-NK patients have focused on FLT3-ITD. In contrast, we focused on FLT3-ITD and FLT3-TKD. We observed that patients with FLT3-TKD mutations tended to have a poorer outcome, although the difference was not statistically significant because of the small sample size. We determined that the presence of FLT3-ITDmut was an independent predictor of poor patient prognosis and that there were no prognostic differences between FLT3-ITD and FLT3TKD mutations. These results suggest the possibility that the presence of FLT3-TKD mutations predicts poor patient prognosis.

Our findings on the hematologic response of patients following induction chemotherapy correlated with the presence or absence of NPM1 and FLT3 mutations. Simi- lar to previous studies, we found that the CR rate was significantly higher in patients with NPM1mut than in patients without NPM1mut. This difference is primarily derived from the greater sensitivity of NPM1mut-containing blasts to the initial chemotherapy [25]. Indeed, we found that patients with non-A-type NPM1mut had superior hematological responses regardless of their FLT3mut status. We observed a similar response in patients with NPM1mut in the absence of FLT3-ITD (table 4).

The treatment protocol for patients with AML is usually dependent on prognostic factors, including cytogenetic results and age. AML patients with NPM1mut/ FLT3-ITDwt, who have a favorable prognosis, are usually treated with conventional chemotherapy, with or without HSCT [26]. Although FLT3mut are regarded as an indication for HSCT, the role of HSCT in patients with NPM1mut and FLT3mut is unclear [26, 27]. We found that HSCT improved survival in AML-NK patients, including patients with NPM1mut/FLT3-ITDmut. However, the OS did not exceed 6 years in patients with NPM1mut/FLT3-ITDmut. Although we did not focus on the detail of HSCT therapy, we found that HSCT improved OS, regardless of the presence of NPM1mut and/or FLT3-ITDmut. In general, patients in favorable risk groups have not been considered candidates for HSCT [28]. We observed that the presence of NPM1mut without FLT3-ITDmut was significantly correlated with a higher CR rate and longer OS and that these patients benefited from HSCT. This study could not provide sufficient evidence to justify the benefit of HSCT because of its small sample size and simple retrospective analysis. Because we observed a favorable impact of HSCT in our study, further studies with a larger sample size are warranted to confirm these findings.

Among patients with NPM1mut, $80 \%$ have A-type NPM1mut, which have a favorable impact on patient prognosis $[17,26]$. The remaining $20 \%$ of patients with NPM1mut have non-A-type NPM1mut. The clinical impact of non-A-type NPM1mut remains unclear. Regardless of the mutation type, mutated NPM1 encodes cytoplasmic NPM, which acts as an oncoprotein [16, 29]. Therefore, non-A-type NPM1mut are believed to have a clinical impact similar to that of A-type NPM1mut. Our results indicate that non-A-type NPM1mut are associated with favorable outcomes. Patients with A-type and nonA-type NPM1mut tended to have more favorable outcomes with no prognostic differences between these two subgroups. Another previous study of 106 patients found that non-A-type NPM1mut (5 patients) were associated with a significantly shorter CR rate and shorter OS com- 
pared with NPM1wt and A-type NPM1mut (13 patients) [7]. However, there was a limitation in that karyotype was not specified for all studied patients. In particular, 1 of the 5 patients with non-A-type NPM1mut were included in the poor-risk group according to karyotype, for 1 of them there was no information about karyotype and 3 of them were included in the standard-risk group without specific karyotypic information.

Interestingly, we found that the interactions between mutations differed according to the type of NPM1mut. Although FLT3mut tended to predict poor prognosis in patients with A-type and non-A-type NPM1mut, their prognostic impact was significant in patients with nonA-type NPM1mut. We included the FLT3-TKD mutation in FLT3mut in our mutational interaction analysis because we observed that the presence of the FLT3-TKD mutation was predictive of poor patient prognosis. Further studies are needed to determine the prognostic interaction between mutations. In addition, our study did not include other molecular markers, such as CEBPA genes, which are known to impact on disease prognosis [3]. Further studies including other prognostic factors are required in the future.

In conclusion, we showed that patients with nonA-type NPM1mut, regardless of FLT3mut status, and patients with NPM1mut in the absence of FLT3-ITD have higher CR rates than patients with NPM1wt and FLT3mut. We found that patients with A-type and nonA-type NPM1mut tended to have better prognoses than patients with NPM1wt and that FLT3-ITD and FLT3TKD mutations were associated with a poor prognosis. The favorable prognostic impact of NPM1mut was shown in the univariate analysis, which evaluated the CR rate and RFS. However, in the multivariate analysis, no prognostic impact of NPM1mut and FLT3mut was detected. Although limited by the small sample size, our study findings on the prognostic interaction between non-Atype NPM1mut and FLT3mut indicate the importance of assessing the subtypes of NPM1mut and FLT3mut in patients with AML-NK.

\section{References}

1 Frohling S, Scholl C, Gilliland DG, Levine RL: Genetics of myeloid malignancies: pathogenetic and clinical implications. J Clin Oncol 2005;23:6285-6295.

- 2 Mrozek K, Heerema NA, Bloomfield CD: Cytogenetics in acute leukemia. Blood Rev 2004;18:115-136.

-3 Vardiman JW, Thiele J, Arber DA, Brunning RD, Borowitz MJ, Porwit A, Harris NL, Le Beau MM, Hellstrom-Lindberg E, Tefferi A, Bloomfield CD: The 2008 revision of the World Health Organization (WHO) classification of myeloid neoplasms and acute leukemia: rationale and important changes. Blood 2009;114:937-951.

4 Thiede C, Steudel C, Mohr B, Schaich M, Schakel U, Platzbecker U, Wermke M, Bornhauser M, Ritter M, Neubauer A, Ehninger G, Illmer T: Analysis of FLT3-activating mutations in 979 patients with acute myelogenous leukemia: association with FAB subtypes and identification of subgroups with poor prognosis. Blood 2002;99:4326-4335.

-5 Thiede C, Koch S, Creutzig E, Steudel C, Illmer T, Schaich M, Ehninger G: Prevalence and prognostic impact of NPM1 mutations in 1485 adult patients with acute myeloid leukemia (AML). Blood 2006;107:4011-4020.
-6 Koh Y, Park J, Bae EK, Ahn KS, Kim I, Bang SM, Lee JH, Yoon SS, Lee DS, Lee YY, Park S, Kim BK: Non-A type nucleophosmin 1 gene mutation predicts poor clinical outcome in de novo adult acute myeloid leukemia: differential clinical importance of NPM1 mutation according to subtype. Int J Hematol 2009;90:1-5.

7 Ahmad F, Mandava S, Das BR: Analysis of FLT3-ITD and FLT3-Asp835 mutations in de novo acute myeloid leukemia: evaluation of incidence, distribution pattern, correlation with cytogenetics and characterization of internal tandem duplication from Indian population. Cancer Invest 2010;28:63-73.

$\checkmark 8$ Bacher U, Haferlach C, Kern W, Haferlach T, Schnittger S: Prognostic relevance of FLT3TKD mutations in AML: the combination matters - an analysis of 3082 patients. Blood 2008;111:2527-2537.

-9 Yamamoto Y, Kiyoi H, Nakano Y, Suzuki R, Kodera Y, Miyawaki S, Asou N, Kuriyama K, Yagasaki F, Shimazaki C, Akiyama H, Saito K, Nishimura M, Motoji T, Shinagawa K, Takeshita A, Saito H, Ueda R, Ohno R, Naoe $\mathrm{T}$ : Activating mutation of D835 within the activation loop of FLT3 in human hematologic malignancies. Blood 2001;97:24342439.

$>10$ Kim HJ: Mutations in AML with a normal karyotype: NPM1 and FLT3-ITD, ready to use as a key prognosticator? Korean J Hematol 2010;45:79-80.
-11 Suzuki T, Kiyoi H, Ozeki K, Tomita A, Yamaji S, Suzuki R, Kodera Y, Miyawaki S, Asou N, Kuriyama K, Yagasaki F, Shimazaki C, Akiyama H, Nishimura M, Motoji T, Shinagawa K, Takeshita A, Ueda R, Kinoshita T, Emi N, Naoe T: Clinical characteristics and prognostic implications of NPM1 mutations in acute myeloid leukemia. Blood 2005; 106:2854-2861.

$\checkmark 12$ Suzuki R, Onizuka M, Kojima M, Shimada M, Okamura K, Fukagawa S, Tsuboi K, Kikuchi A, Kobayashi H, Shintani A, Ogawa Y, Kawada H, Hotta T, Ando K: Prognostic significance of FLT3 internal tandem duplication and NPM1 mutations in acute myeloid leukemia in an unselected patient population. Int J Hematol 2007;86:422-428.

$>13$ Lee JH, Choi SJ, Park JH, Kim H, Joo YD, Lee WS, Zang DY, Kim HJ, Lee KH: Standard induction chemotherapy followed by attenuated consolidation in elderly patients with acute myeloid leukemia. Ann Hematol 2006; 85:357-365.

14 Cheson BD, Cassileth PA, Head DR, Schiffer CA, Bennett JM, Bloomfield CD, Brunning R, Gale RP, Grever MR, Keating MJ, Sawitsky A, Stass S, Weinstein H, Woods WG: Report of the National Cancer Institute-sponsored workshop on definitions of diagnosis and response in acute myeloid leukemia. J Clin Oncol 1990;8:813-819. 
15 Huang Q, Chen W, Gaal KK, Slovak ML, Stein A, Weiss LM: A rapid, one step assay for simultaneous detection of FLT3/ITD and NPM1 mutations in AML with normal cytogenetics. Br J Haematol 2008; 142:489492.

16 Falini B, Mecucci C, Tiacci E, Alcalay M, Rosati R, Pasqualucci L, La Starza R, Diverio D, Colombo E, Santucci A, Bigerna B, Pacini R, Pucciarini A, Liso A, Vignetti M, Fazi P, Meani N, Pettirossi V, Saglio G, Mandelli F, Lo-Coco F, Pelicci PG, Martelli MF: Cytoplasmic nucleophosmin in acute myelogenous leukemia with a normal karyotype. $\mathrm{N}$ Engl J Med 2005;352:254-266.

17 Rau R, Brown P: Nucleophosmin (NPM1) mutations in adult and childhood acute myeloid leukaemia: towards definition of a new leukaemia entity. Hematol Oncol 2009;27: 171-181.

18 Lee KH, Choi SJ, Lee JH, Kim DY, Seol M, Lee YS, Kang YA, Jeon M, Yun SC, Joo YD, Lee WS, Kang MJ, Kim H, Park JH, Bae SH, Ryoo HM, Kim MK, Hyun MS: Clinical effect of reduced-intensity conditioning regimen containing antithymocyte globulin for hematopoietic cell transplantation from unrelated donors. Am J Hematol 2011;86:399405.

19 Boonthimat C, Thongnoppakhun W, Auewarakul CU: Nucleophosmin mutation in Southeast Asian acute myeloid leukemia: eight novel variants, FLT3 coexistence and prognostic impact of NPM1/FLT3 mutations. Haematologica 2008;93:1565-1569.
20 Verhaak RG, Goudswaard CS, van Putten W, Bijl MA, Sanders MA, Hugens W, Uitterlinden AG, Erpelinck CA, Delwel R, Lowenberg B, Valk PJ: Mutations in nucleophosmin (NPM1) in acute myeloid leukemia (AML): association with other gene abnormalities and previously established gene expression signatures and their favorable prognostic significance. Blood 2005;106:3747-3754.

21 Schnittger S, Schoch C, Kern W, Mecucci C, Tschulik C, Martelli MF, Haferlach T, Hiddemann W, Falini B: Nucleophosmin gene mutations are predictors of favorable prognosis in acute myelogenous leukemia with a normal karyotype. Blood 2005; 106:37333739.

22 Dohner K, Schlenk RF, Habdank M, Scholl C, Rucker FG, Corbacioglu A, Bullinger L, Frohling S, Dohner H: Mutant nucleophosmin (NPM1) predicts favorable prognosis in younger adults with acute myeloid leukemia and normal cytogenetics: interaction with other gene mutations. Blood 2005; 106:37403746.

23 Gale RE, Green C, Allen C, Mead AJ, Burnett AK, Hills RK, Linch DC: The impact of FLT3 internal tandem duplication mutant level, number, size, and interaction with NPM1 mutations in a large cohort of young adult patients with acute myeloid leukemia. Blood 2008;111:2776-2784.

24 Schlenk RF, Dohner K, Krauter J, Frohling S, Corbacioglu A, Bullinger L, Habdank M, Spath D, Morgan M, Benner A, Schlegelberger B, Heil G, Ganser A, Dohner H: Mutations and treatment outcome in cytogenetically normal acute myeloid leukemia. N Engl J Med 2008;358:1909-1918.
25 Schneider F, Hoster E, Unterhalt M, Schneider S, Dufour A, Benthaus T, Mellert G, Zellmeier E, Bohlander SK, FeuringBuske M, Buske C, Braess J, Fritsch S, Heinecke A, Sauerland MC, Berdel WE, Buechner $\mathrm{T}$, Woermann BJ, Hiddemann W, Spiekermann K: NPM1 but not FLT3-ITD mutations predict early blast cell clearance and CR rate in patients with normal karyotype AML (NK-AML) or high-risk myelodysplastic syndrome (MDS). Blood 2009; 113:5250-5253.

26 Falini B, Sportoletti P, Martelli MP: Acute myeloid leukemia with mutated NPM1: diagnosis, prognosis and therapeutic perspectives. Curr Opin Oncol 2009;21:573-581.

27 Bornhauser M, Illmer T, Schaich M, Soucek $\mathrm{S}$, Ehninger G, Thiede C: Improved outcome after stem-cell transplantation in FLT3/ ITD-positive AML. Blood 2007;109:22642265, author reply 2265.

28 Dohner H, Estey EH, Amadori S, Appelbaum FR, Buchner T, Burnett AK, Dombret H, Fenaux P, Grimwade D, Larson RA, Lo-Coco F, Naoe T, Niederwieser D, Ossenkoppele GJ, Sanz MA, Sierra J, Tallman MS, Lowenberg B, Bloomfield CD: Diagnosis and management of acute myeloid leukemia in adults: recommendations from an international expert panel, on behalf of the European LeukemiaNet. Blood 2010;115:453-474.

29 Cheng K, Grisendi S, Clohessy JG, Majid S, Bernardi R, Sportoletti P, Pandolfi PP: The leukemia-associated cytoplasmic nucleophosmin mutant is an oncogene with paradoxical functions: Arf inactivation and induction of cellular senescence. Oncogene 2007;26:7391-7400. 